Brit. Heart J., 1965, 27, 116.

\title{
LOCALIZATION OF MONOAMINE OXIDASE IN MAMMALIAN AND REPTILIAN HEART
}

\author{
BY \\ E. MƯlleR* AND A. G. E. PEARSE $\dagger$ \\ From the Department of Pathology, Postgraduate Medical School, London W.12
}

Received December 5, 1963

Numerous studies have been reported concerning the role of monoamine oxidase (MAO) in the metabolism of biogenic amines, especially in the central nervous system and in the heart. A large number of compounds that inhibit MAO have been described, and some of these have been used therapeutically. For this reason we considered that differences in concentration and localization of the enzyme might be of considerable importance in evaluating the results of therapy with MAO inhibitors. Comparison was therefore made between the human heart and those of a number of animals.

\section{MATERIAL AND Methods}

Hearts from both male and female examples of the following species were used in this study: frog (Rana temporaria), mouse, rat, guinea-pig, rabbit, cat, dog. Human hearts were obtained at necropsy, as soon as possible after death. In the case of the animals, the hearts were removed immediately after death and broad slices were quenched at $-70^{\circ} \mathrm{C}$. and then cut in the cryostat at $12 \mu$. It is possible to use thinner sections than this but at least $8 \mu$ is required to obtain adequate contrast.

The method used for demonstration of MAO was a simple modification of that of Glenner, Burtner, and Brown (1957), using tetra-nitro BT in place of nitro BT. The incubation medium contained: $25 \mathrm{mg}$. trypta-

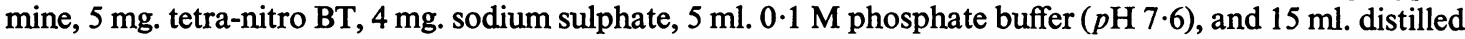
water.

After incubation for 20 to 90 minutes at $37^{\circ} \mathrm{C}$. the sections were washed in distilled water, counterstained with 2 per cent aqueous methyl green (chloroform-washed) and mounted in glycerine jelly. Adjacent serial cryostat sections were stained for glycogen by Bauer's method after post-fixation in cold Bouin's fluid.

From the series of 5 human hearts ( 3 adult, 2 stillborn infants) a standard selection of 6 blocks was taken. These were processed as described above.

\section{RESULTS}

The most striking differences in localization of MAO were found in the rat heart (Fig. 1 and 2A). The gross distribution of the enzyme was characteristic and reproducible. It was present throughout the whole heart but activity in the left ventricle was always considerably stronger than that of the right ventricle. The region of the left apex (Fig. 1) was constantly more active than the surrounding areas of myocardium, and another region of high activity could be seen at the base of the heart (Fig. 1). This was assumed to be related to the presence in this region of parts of the conducting

* Present address: Geigy A. G., Basel, Switzerland.

$\dagger$ In receipt of a grant from the British Heart Foundation. 
system (sino-atrial and atrio-ventricular nodes). The activity of the endocardial region was characteristically strong (Fig. 1 and 2A) and only part of this high activity could be attributed to the fibres of the conducting system.

An essentially similar pattern was noted in the adult human heart. Fig. 3 shows the reaction in human left ventricle. Activity is largely confined to the sarcoplasmic reticulum but it is especially high in relation to the mitochondrial accumulations around the sarcolemmal nuclei. No activity could be detected in the hearts of the two stillborn infants.

In the guinea-pig, though the over-all reaction was much weaker, its pattern resembled that seen in the rat and human heart. The strongest reaction in this animal was in the endocardium of the left ventricle, in relation to the fibres of the conducting system (Fig. 4A). In man and in the rat the contrast between the activity of the myocardium and that of the conducting system was less striking. The high level of glycogen in the Purkinje fibres, long known and used for their differentiation from muscle fibres, is shown in Fig. 4B.

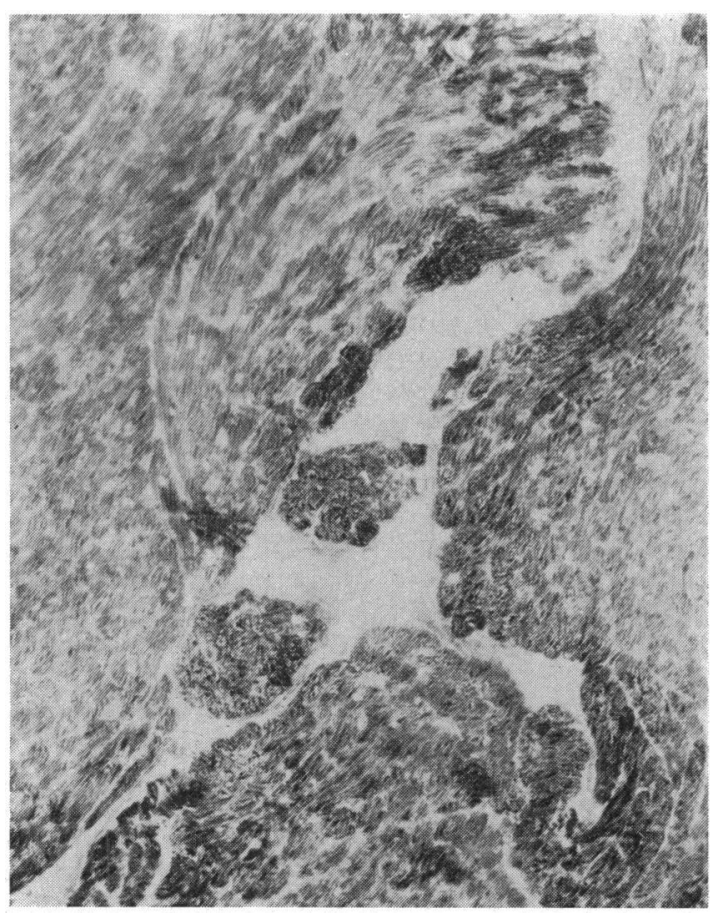

A

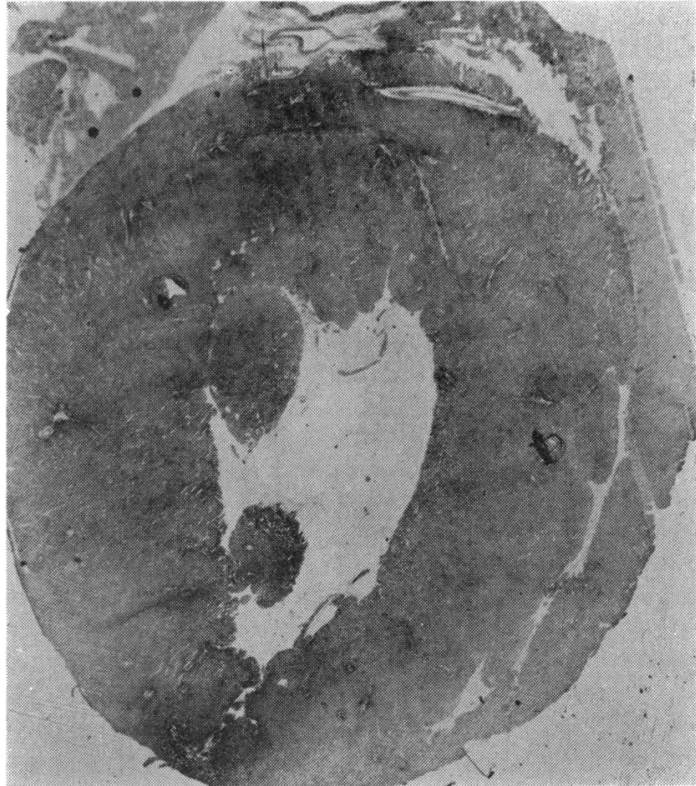

Fig. 1.-Whole rat heart: right, right ventricle; left, left ventricle. Highest MAO activity on left side and also at the base and at the apex on this side. $(\times 8 \cdot 25$.

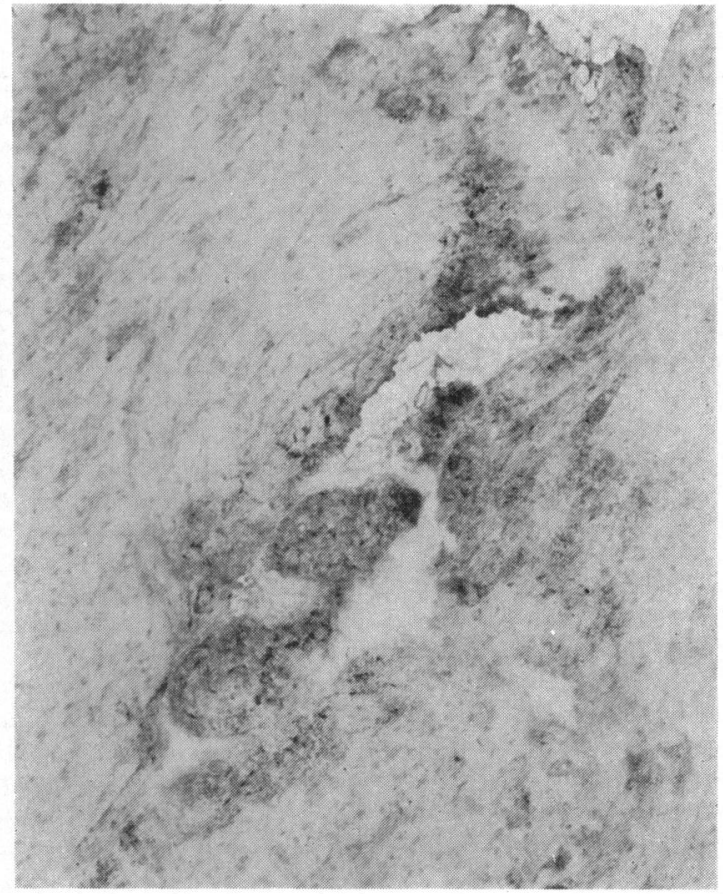

B

Fig. 2.-Rat heart (left ventricle). (A) MAO activity especially strong in subendocardial region. (B) High glycogen content of subendocardial fibres. $(\times 150$.) 


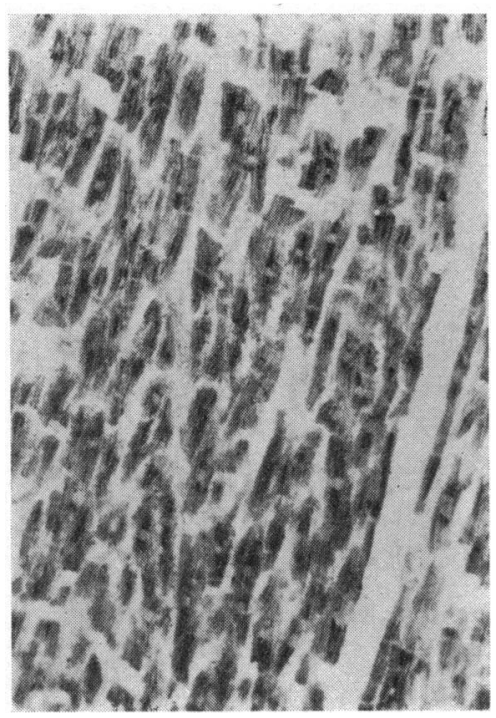

FIG. 3.-Human myocardium (left ventricle). Shows MAO activity in sarcoplasmic reticulum and, especially, around sarcolemmal nuclei. $(\times 127$.

\section{MÜLLER AND PEARSE}

A constantly positive relation between glycogen and MAO was noted in all species examined. This was not confined to the conducting system (Fig. $2 \mathrm{~A}$ and $\mathrm{B}, 4 \mathrm{~A}$ and $\mathrm{B}$ ) for even in those animals where MAO was practically absent positive regions of myocardium showed high glycogen content.

Hearts from the other four mammals (mouse, rabbit, cat, dog) gave a generally weak reaction; whole regions apparently lacked the enzyme altogether. In all species MAO activity was greater in the male than in the female.

The frog heart was exceptional in that here the whole myocardium showed an intense reaction, almost equal in all areas. The reaction product, as in the case of mammalian hearts, was located in the sarcoplasmic reticulum between the muscle fibres (Fig. 5).

\section{Discussion}

The results presented here offer confirmation of a number of biochemical observations including those of Bernheim and Bernheim (1945), who first showed that MAO activity in rat heart was much higher than in the cat and dog. They also confirm the results of Zeller (1961) who described both the sex variation and the increase of MAO with age. The absence of

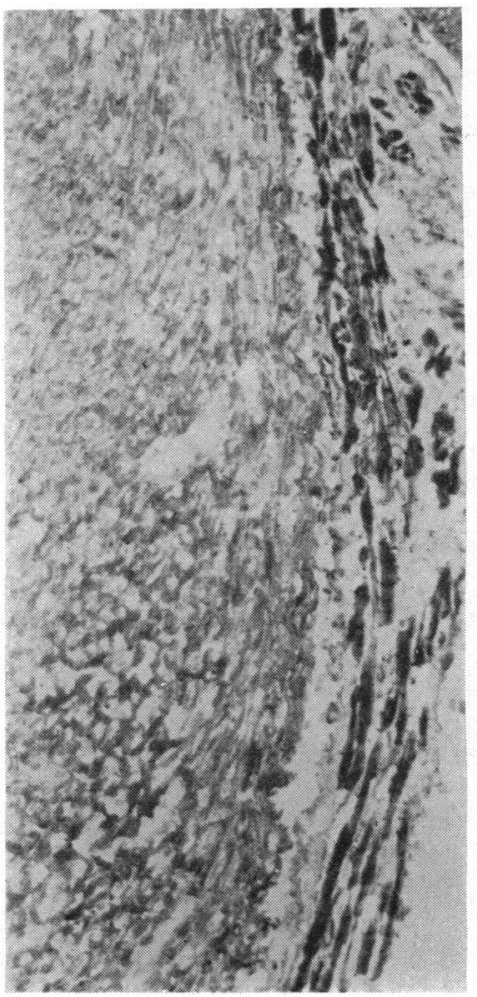

A

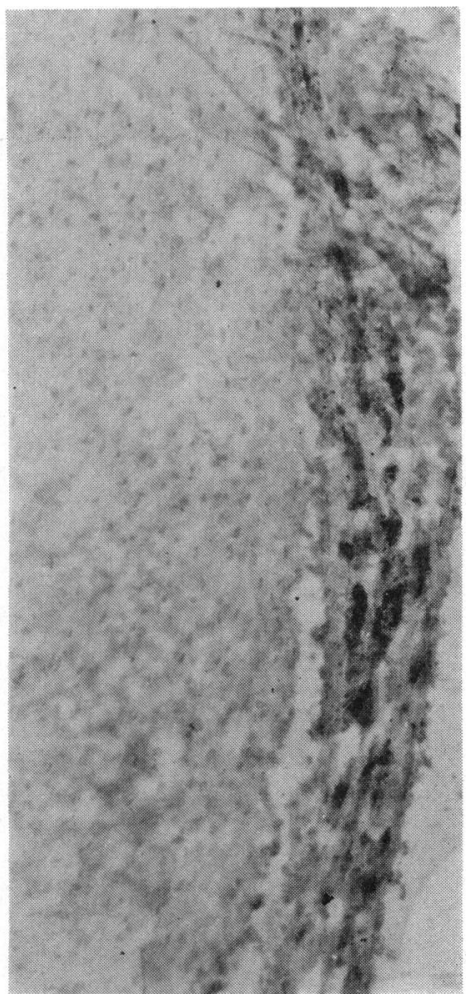

B

FIG. 4.-Guinea-pig heart (left ventricle). (A) Shows high MAO activity and (B) shows high glycogen levels in the subendocardial fibres. $(\times 100$. 


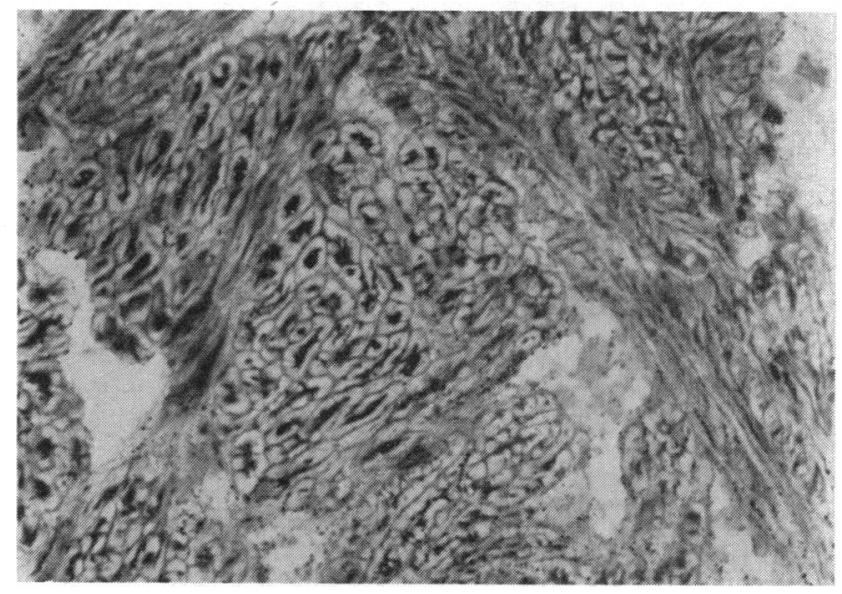

FIG. 5.-Frog myocardium. High MAO activity in the sarcoplasmic reticulum. $(\times 370$.)

MAO from newborn rat hearts was specifically noted by Novick (1961) who showed that enzyme activity could be recorded only at the third week of life. Skillen, Thienes, and Strain (1962) determined MAO level in rats' hearts and showed that the amount in normal males was significantly greater than in normal females.

In addition to our confirmation of the biochemical findings recorded above, we must consider the implications of the different levels of MAO in the left and right heart and the high levels observed in the conducting system.

If we accept the premise of Zeller (1961) that tyramine, tryptamine, serotonin, and the catecholamines are physiological substrates of MAO it follows that differences in level of MAO may be reflected in differences of catecholamine level. On the other hand it is equally possible that areas with high rates of production of catecholamines may be provided with the necessary high levels of MAO for their destruction. Control of catecholamine levels may in turn control the levels of phosphorylase and thus of glycogen. Recent work by Angelakos, Fuxe, and Torchiana (1963) on the distribution of catecholamines in the mammalian heart has shown that in the rabbit and guinea-pig noradrenaline levels are significantly higher on the right side; in particular the right ventricle shows the highest levels. The fact that our results offer the converse of these findings suggests that high MAO levels, in effect, produce low catecholamine levels and vice versa, as would be expected.

Therapeutically the effects of MAO inhibitors should be manifest first in regions of low MAO activity. Ultimately, however, the results might well be stronger in those regions where MAO activity is most important biologically. These may be the regions where it is most active. Little seems to be known about the relative importance of MAO and other enzyme systems such as catechol O-methyl transferase (Axelrod et al., 1958) in the destruction of biogenic amines.

When we come to consider the strong MAO activity of the conducting system we are confronted with a paradox. This concerns the occurrence of high MAO and high cholinesterase (ChE) levels in the same site. The idea of a reciprocal relation between the two enzymes, first presented 20 years ago by Langemann, Roulet, and Zeller (1943), has generally been accepted. Nevertheless the high activity of $\mathrm{ChE}$ in the conducting systems of mammalian hearts must also be accepted (Carbonell, 1956). The anomaly of high MAO levels, usually associated with adrenergic mechanisms, in this specialized system may be explicable on the basis of precise localization. According to Carbonell, ChE activity is confined to the myofibrils of the special conducting cells, whereas MAO is certainly situated in the surrounding sarcoplasmic reticulum and its mitochondria (sarcosomes). These locations would allow the myofibril to respond to cholinergic stimuli while being protected by 
the surrounding MAO activity from the influence of catecholamines, circulating or produced in situ.

\section{SUMmaRY}

A modified tetrazolium method has been used to demonstrate the sites and levels of monoamine oxidase (MAO) activity in the human heart and in hearts from frogs, mice, rats, guinea-pigs, rabbits cats, and dogs.

In three species (rat, man, and guinea-pig) pronounced differences between right and left heart were observed. Highest MAO levels were invariably found on the left side.

A high concentration of MAO was observed in association with the conducting system (node, bundle, and subendocardial fibres).

The significance of these findings is discussed.

\section{REFERENCES}

Angelakos, E. T., Fuxe, K., and Torchiana, M. L. (1963). Chemical and histochemical evaluation of the distribution of catecholamines in the rabbit and guinea pig hearts. Acta physiol. scand., 59, 184.

Axelrod, J., Inscoe, J. K., Senoh, S., and Witkop, B. (1958). O-methylation, the principal pathway for the metabolism of epinephrine and norepinephrine in the rat. Biochim. biophys. Acta (Amst.), 27, 210.

Bernheim, F., and Bernheim, M. L. C. (1945). The inactivation of tyramine by heart muscle in vitro. J. biol. Chem., 158, 425.

Carbonell, L. M. (1956). Esterases of the conductive system of the heart. J. Histochem. Cytochem., 4, 87.

Glenner, G. G., Burtner, H. J., and Brown, G. W., Jr. (1957). The histochemical demonstration of monoamine oxidase activity by tetrazolium saltş. J. Histochem. Cytochem., 5, 591.

Langemann, H., Roulet, F., and Zeller, E. A. (1943). Fermentuntersuchungen an gesunden und tuberkulösen menschlichen Lungen. Klin. Wschr., 22, 644.

Novick, W. J., Jr. (1961). The effect of age and thyroid hormones on the monoamine oxidase of rat heart. Endocrinology, 69, 55.

Skillen, R. G., Thienes, C. H., and Strain, L. (1962). 5-hydroxytryptamine, 5-hydroxytryptophan decarboxylase and monoamine oxidase in hearts of normal, thyroid-fed and propylthiouracil-fed male and female rats. Endocrinology, 70, 743 .

Zeller, E. A. (1961). Monoamine oxidase "Monoamines et système nerveux central" Symp. Bel-Air, Geneva, Sept 1961. Georg, Geneva. 